welding of different races and religions and the attempt to create a corporate college spirit has been astonishingly successful. There is evidence of goodwill from all races, and perhaps the most disappointing feature is the comparatively small number of European students who have enrolled. This is partly accounted for by the fact that pupils in the European schools already take the Cambridge Higher School Certificate examination. Nevertheless, it is believed that when the standards of the College, both academic and cultural, become better known, a higher representation of the European section of the community will be obtained.

The normal academic and social attributes of a residential college are in a formative state. Full use has been made of the limited area available for sports and a Student Society has been formed, to which are affiliated various student clubs. 'There is a students' refectory in the main block, a senior common room is in existence, and the first phase of the library is completed, containing 8,000 volumes and 3,000 periodicals.

An introductory term began on April 23, 1956, during which, on July 12, 1956, the Vice-President of India, Dr. Radhakrishnan, opened the part of the College which has been designated the Gandhi Memorial Academy. Himself a philosopher of high repute, the Vice-President delivered a memorable address concerning the vast problems and responsibilities of education in East Africa.
During the first term of the College year proper, which began on October 1, 1956, the College was honoured by a visit from H.R.H. Princess Margaret, who performed the formal opening ceremony of the whole College on October 24. In her speech, the Princess referred to the importance of a college of this nature to territories the activities of which, whether agricultural, commercial or industrial, are increasing so rapidly. Princess Margaret said that the College had a twofold task: first, to produce men and women trained in as specialized and practical field; and secondly, to send out into the world people with wide interests who have, by their association with their fellow-students, been set firmly on the road leading to wise eitizenship. Princess Margaret showed a warm and lively regard for all aspects of life and work at the College and spoke to several of the students about their own problems and interests.

The Royal Technical College, having been launched thus happily and auspiciously, and the eagerness for work and the goodwill of the first entry of students having been displayed so encouragingly, the staff of the College faces the future of this farsighted enterprise with confidence. Their only anxiety is that this project, so warmly greeted by the young people of East Africa and so vital to the development of their country, will not be checked or impaired by lack of financial support for its urgently needed capital development.

\title{
CRYSTAL DYNAMICS
}

D URING the second week of January the Physical Society held a two-day Conference at Queen Mary College, London, on "Crystal Dynamics". There was a large attendance to hear more than twenty contributions from many leading British physicists. $\mathrm{It}$ is an indication of the growth of interest in the theory of solids that a whole conference could be devoted so successfully to such a relatively specialized branch of the subject. Many of the topics that were dealt with have long histories such as the specific heat of crystals associated with lattice vibrations, and the thermal expansion resulting from anharmonicity. Other fields discussed were entirely unknown before the Second World War, for example, the determination of frequency spectra by means of neutron diffraction. In yet a third group of topies old problems were dealt with on which new light has been shed in recent years by extensive experimental and theoretical research. An example of this group is electron-phonon interaction which, in some circumstances, dominates the electrical and thermal conductivity of metals at low temperatures.

The Conference was divided into four sessions, each of which was opened by a lecture in which the subject of the session was reviewed, thus placing the audience in a position to appreciate the shorter specialized contributions, describing recent work, which followed.

The first session was opened by Dr. M. Blackman (Imperial College of Science and Technology, London), who reviewed the types of vibrational spectra of crystals which have been obtained theoretically, and the heat capacity curves deduced from them. The Debye $T^{3}$ law was discussed from the theoretical and experimental point of view and it was pointed out that only in one or two cases was it really certain that the $T^{3}$ region had been reached in experimental measurements.

An interesting contribution came from Prof. M. H. L. Pryce (Bristol), who dealt with a new form of lattice statics. He reported calculations which have been carried out to determine atomic displacements caused by defects such as vacancies, interstitial atoms, impurity atoms and dislocations. The method uses Fourier transforms, as in the determination of the normal modes of the lattice. It has the advantage that the discrete character and the elastic anisotropy of the crystal are automatically taken care of. The results are promising and should be better than calculations based on an elastic theory of a continuum supplemented by the localized relaxation of a fow atoms.

In a short contribution Prof. C. Domb (King's (ollege, London) dealt with the calculation of the anharmonic properties of a solid such as thermal expansion and compressibility. A simple attempt at dealing with this problem was made by assuming a Debye model in which the $\theta$-value was dependent on volume. However, the results did not agree well with experiment. For an extremely anharmonic solid, like helium, there was marked disagreement with experiment. Even for solid argon, recent experimental results obtained at Queen Mary College differed appreciably from theory. Accordingly, a different approach due to Henkel, which is a generalization of the Einstein model for a harmonic solid, was adopted. The results were in surprisingly good agreement with experiment when a Isennard-Jones 6,12 potential was used. 
In a contribution entitled "Solid Argon as a Model Substance", Dr. E. R. Dobbs (Queen Mary College, London) surveyed experimental techniques used to obtain accurate measurements of the expansivity, compressibility and specific heat of poly-crystalline argon. A comparison with the theoretical calculations of Domb and Zucker showed that their assumption of anharmonic lattice vibrations in a modified Einstein model was in agreement with the experimental results for expansivity and compressibility but, not unexpectedly, failed to account for the variation of the specific heat at low temperatures.

Another contribution to the first session was made by Dr. D. Bijl (St. Andrews), who pointed out that the customary method of using a temperaturedependent or effective Debye temperature has serious disadvantages. A better method is to plot the ratio between the measured specific heat and the Debye specific heat calculated with a characteristic temperature obtained from the true $T^{3}$ region.

The second session, on "Vibrational Spectra", was opened by Dr. G. L. Squires (Cambridge), who discussed the method by which the frequencies of the normal vibrations of a crystal may be determined. These frequencies can be determined by measuring the momentum changes of slow neutrons which have been scattered inelastically by the crystal. A neutron excites or de-excites a phonon in a crystal lattice, and the resulting change of energy, being of the same order as the initial energy, was readily measurable. The inelastic scattering of $\mathrm{X}$-rays was said to be not so suitable for measuring phonon frequencies because the change in the energy of the $\mathrm{X}$-ray quantum was of the order of $10^{-5}$ of its initial energy. Such small changes in energy are not at present detectable and the phonon frequencies havé to be deduced from intensity measurements. So far, owing to the comparative weakness of neutron sources, most of the measurements of phonon frequencies have come from $X$-ray experiments, but as high-flux reactors, such as DIDO at Harwell, become available it was expected that the neutron method would be used increasingly.

Dame Kathleen Lonsdale (University College, London), in a short contribution, discussed methods of determining the anisotropic vibrations in crystals. Diffraction methods enabled one to determine the values of the mean square amplitudes of individual ions or atoms in different directions relative to the crystal axes. In the case of molecular crystals it was of particular interest to distinguish thermal movements of the molecules relative to each other from intra-molecular vibrations. There are several different ways of approaching the problem : diffuse scattering on photographs of stationary crystals; coherent scattering of neutrons or X-rays leading to a Fourier analysis; or measurements at varying temperatures. The first gave much information, but at present could not be interpreted except in the case of very simple and symmetrical structures. The second is the standard method. The third has provided useful confirmation of the results obtained and gives promise of leading to good estimates of zero-point energies.

Dr. D. H. Martin (Queen Mary College) outlined the need for information about the absorption spectra of ionic crystals in the very far infra-red, and discussed the feasibility of observing such spectra with good results by making use of the super-conducting bolometer.

An interesting theoretical contribution was made by Dr. G. V. Chester (Birmingham), who dealt with inelastic neutron scattering in crystals. The theory of the coherent inelastic scattering of neutrons by a single crystal showed that, for every process in which the neutron absorbs or emits a single phonon, the neutrons are scattered in all directions, and the intensity of scattered neutrons in any given direction shows sharp peaks when plotted as a function of energy. Because of mutual scattering and other effects, phonons had only a finite lifetime and, therefore, their energies were not precisely defined in accordance with the Uncertainty Principle. This resulted in a broadening of the peaks in the neutron diffraction pattern. From the line width it was possible to determine the total scattering crosssection for a phonon both as a function of wave number and polarization. It was thought that the main applications of this method would lie in the study of phonon-phonon and electron-phonon scattering.

The third session, at which the chair was taken by Prof. R. E. Peierls, was opened by a review of thermal conductivity given jointly by Dr. J. M. Ziman (Cambridge) and Dr. R. Berman (Oxford). Dr. Ziman considered the theory of the various effects which led to thermal resistance in crystals : interstitial atoms ; vacancies; substitutional impurities; dislocations; stacking faults; and surface roughness. He was able to show what might be expected for the temperaturedependence of the thermal conductivity due to each of these causes. Dr. Berman dealt with experimental data relating to the thermal conductivity of dielectrics at very low temperatures and was able to correlate this data with the theoretical predictions previously discussed by Dr. Ziman.

In a short contribution Dr. H. M. Rosenberg (Oxford) described experimental results concerning the electronic thermal conductivity of the metals zinc and cadmium. He was able to relate the observations to the known variation of the $\theta_{D}$-values with temperature in the region of very low temperatures.

Dr. J. Wilks (Oxford) described experiments on the thermal boundary resistance between metals and liquid helium II which showed that the dependence of this resistance on temperature is similar to that of the thermal contact resistance between two solids. Theory predicts a heat transfer which varies as $T^{3}$, and which depends on the properties of the metal and the liquid helium. Agreement with experiment is far from satisfactory ; the observed dependence on temperature is nearer to $T^{2}$. It was suggested that this discrepancy may be due to the theoretical treatment, which assumes that the media are continuous.

Two other short contributions were made by Dr. R. Fürth (Birkbeck College, London), who spoke on the theory of fluctuations of strain and stress in crystals, and by Dr. E. H. Sondheimer (Queen Mary College), who discussed a point hitherto overlooked in the theory of the thermal conductivity of metals in strong magnetic fields.

The final session, with Prof. N. F. Mott in the chair, was opened by Prof. Harvey Brooks (Harvard), who reviewed the theory of electron-phonon interaction in metals. A searching analysis was given of the various attempts which have been made to calculate the matrix of the scattering potential for electrons in metals. Particular attention was paid to the selfconsistent method of Bardeen and the more recent method of the deformation potential. An interesting point which emerged was that, even at what are 
normally considered low temperatures, Umklappprocesses are frequent in metallic sodium, and that at room temperatures such processes account for some 90 per cent of the resistivity.

Prof. G. O. Jones (Queen Mary College) summarized the work which has been carried out on super-conductivity at pressures of 10,000 atmospheres and above. Although the electron-phonon interaction theory of superconductivity, developed by Fröhlich and Bardeen, had appeared to account more or less exactly for the change in transition temperatures with change in isotopic mass, the effect of varying the volume is by no means straightforward. For metals which would be regarded as normal with respect to their ordinary electrical properties under high pressure, having a resistivity which varies approximately as $V^{2 \gamma}$, where $\gamma$ is the Grüneisen constant, the superconducting behaviour varies enormously. In some cases the transition temperature varies according to expectation as $V \gamma$, but in others the variation is more nearly proportional to $V^{\mathbf{4}} \gamma$, and in one case (thallium) there is a reversal in the sense of the variation at about 1,000 atmospheres.
In a short contribution, Dr. P. G. Klemens (Sydney) spoke of the conduction properties of monovalent metals at very low temperatures and explained a discrepancy which appears to exist between thermal and electrical conductivity. Dr. A. B. Pippard (Cambridge) in a final contribution dealt with acoustic absorption in metal crystals. He described how an analysis, by standard methods of conduction theory, of the attenuation of a longitudinal ultrasonic wave shows that, so long as the electronic free path is shorter than the wave-length, the attenuation varies as the square of the frequency, but, when the free path is long compared with the wave-length, the attenuation is proportional to the frequency and independent of free path. The transition region has been investigated by Bömmel and found to show qualitative agreement with theory. The result for a long free path is identical with that derived by the arguments used to describe electron-phonon interaction in quantum theory of conduction. It seems likely, then, that the result derived 'classically' by consideration of electron trajectories is valid over the whole range of free paths.

H. JoNES

\section{OBITUARIES}

\section{Dr. J. H. Partridge}

John Henry Partridge, a senior member of the Research Laboratories of the General Electric Company, Ltd., died on November 30, 1956, at the age of fifty-three. For many years Partridge had been head of the department of the Laboratories concerned with glass, refractories and ceramics. He was a man who loved to do things with his own hands and he was always anxious to translate, as rapidly as possible, laboratory findings into works' application. He was very frequently to be found in the works both of his own Company and of other firms in the glass industry, giving his help generously. $\mathrm{He}$ had suffered from a heart attack some six years ago, and the restraint which his medical adviser attempted to impose upon him he found very hard to bear. It was very difficult to stop him trying to carry out a full programme of works' visits and society meetings, when in the interest of his health he should at times have been taking life more quietly. His ill-health never interfered with his great sense of fun, and he was a delightful colleague with whom to travel either to a works of the Company or to some international congress.

Partridge was a most conscientious man, and this virtue was reflected in his record of service to scientific and technical societies. He was a member of the Council of the Society of Glass Technology for several periods since 1934, and was honorary secretary of the Society during 1946-49. At the time of his death he was in office as president, having been elected in April 1956; election to this office was an honour which Partridge deeply appreciated, and he had undertaken the duties with characteristic energy. $\mathrm{He}$ was a member of the Ceramic Society and a Founder Fellow of the Institute of Ceramics; he had travelled much on the Continent to attend international congresses on glass.

In the G.E.C. Laboratories he had done a great deal of work on the development of new glasses and refractories; he was the author of two monographs, one on "Refractory Materials for the Glass Industry" and the other on "Glass-to-Metal Seals". Ho had written many papers on these and related subjects.

Dr. Partridge was educated at King Edward VI Grammar School, Birmingham, and in 1921 he entered the School of Metallurgy in the University of Birmingham, and in due course he obtained the degree of Ph.D. During the period 1925-26 he was awarded a grant from the Department of Scientific and Industrial Research, and a Carnegie Scholarship from the Iron and Steel Institute. In 1947 he received his D.Sc. for his contribution to scientific and technological knowledge in the fields of glass and refractories.

His colleagues in the General Electric Company and a much wider circle of colleagues in the glass industry in Britain and abroad will feel keenly the loss of a man whose enthusiasm and good humour had endeared him to them-a man whose enjoyment of life was so infectious. His friends will have great sympathy for his wife and three children.

R. W. Douglas

\section{Prof. Joseph Varga}

Prof. Joseph VARGA, a member of the Hungarian Scientific Academy and professor of chemical technology in the Technical University in Budapest, died at the age of sixty-five on December 28. He received his degree in chemical engineering in 1908 at the Technical University in Budapest, and then he was appointed assistant there. In 1920 he was appointed Privatedocent, and in 1923 professor in the Institute for Chemical Technology. He worked in the field of the hydrogenation of coal and coal-tars and made several valuable contributions to the scientific problems involved and industrial applications. Arguing with Bergius-who first liquefied coal-he proved by classical experiments that the hydrogenation of coal is 